\title{
Dielectric and Conductivity Anisotropy in Liquid Crystalline Phases of Strongly Polar Thioesters
}

\author{
A. MikulKo ${ }^{a, *}$, M. Fraśs ${ }^{a}$, M. Marzec $^{a}$, S. Wróbel ${ }^{a}$, \\ M.D. OssowsKa-Chruściel ${ }^{b}$ AND J. ChruŚCIeL ${ }^{b}$ \\ ${ }^{a}$ Institute of Physics, Jagiellonian University \\ Reymonta 4, 30-059 Kraków, Poland \\ ${ }^{b}$ Institute of Chemistry, University of Podlasie \\ 3-go Maja 54, 08-110 Siedlce, Poland
}

Dedicated to Professor Jerzy Janik on the occasion of his 80th birthday

The main objective of this paper is to study dielectric properties of two compounds of acronyms $5 \mathrm{OSCl}$ and $6 \mathrm{OSCl}$ having strongly polar bond $(\mathrm{C}-\mathrm{Cl})$ at para position. Dielectric measurements were done in the frequency range from $40 \mathrm{~Hz}$ to $15 \mathrm{MHz}$. The dielectric spectra were measured for two principal alignments. For homeotropic alignment, the reorientation of molecule around the short molecular axis was observed. Both substances studied exhibit large positive dielectric anisotropy and the anisotropy of conductivity was obtained in smectic A as well as nematic phase.

PACS numbers: 61.30.-v, 61.30.Eb, 77.22.-d, 66.10.Ed

\section{Introduction}

Since the liquid crystal displays play an important role in computer technology, new liquid crystalline materials showing high dielectric permittivity and anisotropy are required. For that reason, measurements of dielectric permittivity tensor principal components: $\varepsilon_{\perp}^{\prime}(\omega)$ and $\varepsilon_{\|}^{\prime}(\omega)$ as well as complex ionic conductivity $\sigma$ are of the great importance [1-5]. Before a material can be selected for applications one should study its frequency, bias field, measuring field and temperature characteristics.

*corresponding author; e-mail: adriana.mikulko@uj.edu.pl 
(a)

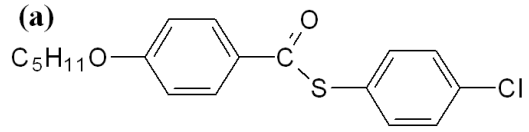

Heating: $\mathrm{Cr} 76.6^{\circ} \mathrm{C}$ N $85.6^{\circ} \mathrm{C}$ I

Cooling: I $86.4^{\circ} \mathrm{C} N 78.2^{\circ} \mathrm{C}$ SmA $56.7^{\circ} \mathrm{C} \mathrm{Cr}$

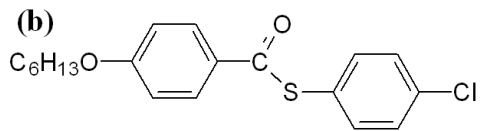

Heating: $\mathrm{Cr} 64.9^{\circ} \mathrm{C} \operatorname{SmA} 86.1^{\circ} \mathrm{C} \quad \mathrm{N} 90.7^{\circ} \mathrm{C} \mathrm{I}$

Cooling: I $91.4^{\circ} \mathrm{C} \mathrm{N} 84.7^{\circ} \mathrm{C} \operatorname{SmA} 46.1^{\circ} \mathrm{C} \mathrm{Cr}$

Fig. 1. Molecular structure and phase sequence obtained for $5 \mathrm{OSCl}$ (a) and $6 \mathrm{OSCl}$ (b).

In scope of this paper, two compounds: $4^{\prime}$-chlorophenol-4- $n$-alkoxythiobenzoates (5OSCl and $6 \mathrm{OSCl}$ ) having strongly polar bond $(\mathrm{C}-\mathrm{Cl})$ at para position [6] have been studied. Their molecular structures and phase sequences obtained on heating and cooling are presented in Fig. 1.

\section{Experimental}

Dielectric measurements were done by using Agilent 4294A impedance analyzer in the frequency range between $40 \mathrm{~Hz}$ and $15 \mathrm{MHz}$ for two principal alignments of the samples. The measurements were performed during heating and cooling of the samples. Temperature was controlled by Eurotherm 2604, and measured by a thermocouple attached to Agilent 34401A multimeter controlled by a computer. The computer with adequate software that enables data acquisition, transferring, and displaying interesting material parameters such as dielectric permittivity, conductivity, loss factor, and other, controlled the experiment. The samples were put in three different types of the gold-coated $5 \mu \mathrm{m}$ cells, namely the planar cell $(\mathrm{Au}-\mathrm{HG})$ and homeotropic cell $(\mathrm{Au}-\mathrm{HT})$ and the $(\mathrm{Au})$ cell with no surface treatment. Planar and homogeneous alignment was obtained by slowly cooling of the sample in Au-HG cells from the $\mathrm{N}$ to $\mathrm{SmA}$ phase. On the other hand, homeotropic alignment in nematic and smectic phase was obtained by applying a DC bias electric field of the order of $10^{6} \mathrm{~V} / \mathrm{m}$ to the sample planar aligned in $\mathrm{Au}$ cells.

\section{Results and discussion}

Dielectric measurements were done for two principal alignments of substances studied to obtain parallel and perpendicular components of the electric permittivity. For homeotropic alignments a strong dielectric relaxation process was found in the SmA phase for both substances (Fig. 2). A Cole-Cole function with conductivity term (Eq. (1)) was fitted to the experimental data

$$
\varepsilon^{*}(\omega)=\varepsilon(\infty)+\frac{\Delta \varepsilon_{0}}{1+\left(\mathrm{i} \omega \tau_{\mathrm{R}}\right)^{1-\alpha}}-\mathrm{i} \frac{\sigma(\omega)}{\varepsilon_{0} \omega},
$$

where $\varepsilon(\infty)$ is a high frequency electric permittivity, $\varepsilon_{0}$ - electric permittivity of the free space, $\Delta \varepsilon_{0}$ - dielectric strength, $\tau_{\mathrm{R}}$ - relaxation time, $\alpha$ - distribution parameter of relaxation times $(0 \leq \alpha \leq 1), \sigma(\omega)$ - ionic conductivity, and 


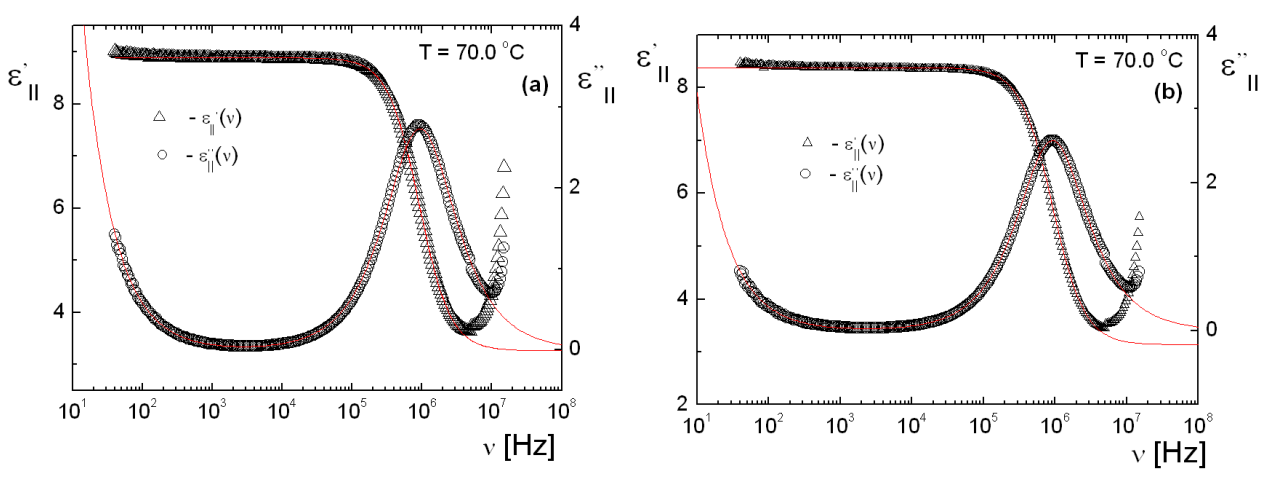

Fig. 2. Dielectric dispersion and absorption versus frequency $(\nu)$ obtained for homeotropic alignment in $\mathrm{SmA}$ phase for $5 \mathrm{OSCl}$ (a) and $6 \mathrm{OSCl}(\mathrm{b})$.

TABLE

Fitting parameters obtained for both substances at temperature of $70.0^{\circ} \mathrm{C}$.

\begin{tabular}{c|c|c|c|c|c}
\hline \hline Substance & $\Delta \varepsilon_{0 \|}$ & $\varepsilon_{\infty \|}$ & $\nu_{\mathrm{R} \|}[\mathrm{MHz}]$ & $\alpha$ & $\sigma_{\|}[\mathrm{S} / \mathrm{m}]$ \\
\hline $5 \mathrm{OSCl}$ & 5.63 & 3.26 & 0.910 & 0.012 & $3.21 \times 10^{-9}$ \\
$6 \mathrm{OSCl}$ & 5.25 & 3.13 & 0.917 & 0.014 & $1.79 \times 10^{-9}$
\end{tabular}

$\omega=2 \pi \nu$ is a circular frequency. The fitting parameters are presented in Table for one temperature. Solid lines in Fig. 2a and b are theoretical fits of Eq. (1).

The activation energy calculated for this process is close to $100 \mathrm{~kJ} / \mathrm{mol}$ for both substances studied and is characteristic of the reorientation of molecule around the short molecular axis [7]. In both cases the $\alpha$ parameter is very small. This means that the relaxation process observed is a single Debye-type process.

Perpendicular and parallel components of the static electric permittivity are presented in Fig. 3. To obtain homeotropic alignment of the sample in Au-HG and $\mathrm{Au}$ cells the bias field was applied in the nematic phase. The homeotropic alignment of the SmA phase was obtained by slowly cooling of the aligned N phase. As seen for cells with planar alignment $(\mathrm{Au}-\mathrm{HG})$ the electric field applied was less effective than surface interaction in Au-HT cells to obtain the homeotropic state. It is worth noting that the parallel component of electric permittivity is distinctly higher than perpendicular one. Moreover, there is a step at the transition from the nematic to the smectic A phase and both components of electric permittivity have a lower value in the $\mathrm{SmA}$ phase. Such behavior shows that $\mathrm{SmA}$ phase is a $\mathrm{SmA}_{2}$ one showing antiferroelectric order, because decrease in $\varepsilon_{\|}(T)$ with temperature lowering is connected with short range dipole-dipole negative correlation of the molecules [7-10]. As seen in Fig. 3 in the case of nematic phase, Au-HT cells give very good alignment. However, upon the $\mathrm{N}-\mathrm{SmA}$ phase transition the homeotropic alignment is lost. 

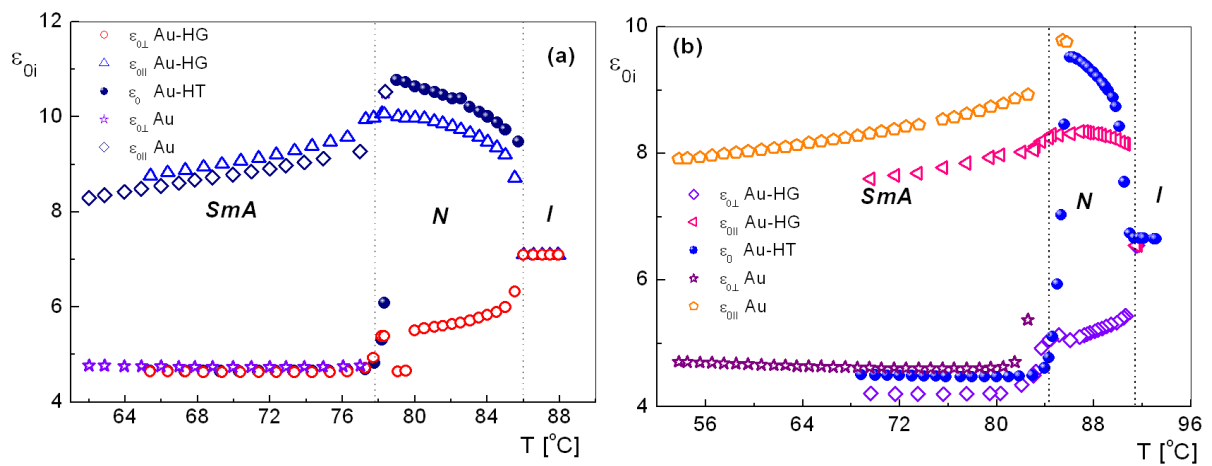

Fig. 3. Static electric permittivities versus temperature obtained for planar and homeotropic alignment for $5 \mathrm{OSCl}$ (a) and 6 $\mathrm{OSCl}(\mathrm{b})$ in the $\mathrm{N}$ and $\mathrm{SmA}$ phases.

Dielectric anisotropy is defined as $\Delta \varepsilon=\varepsilon_{\|}-\varepsilon_{\perp}$. It is worth noting that the dielectric anisotropy is positive in nematic as well as in smectic A phase for both substances studied (Fig. 3). Due to the large and positive dielectric anisotropy, one can observe a nice Fréedericksz transition from planar to homeotropic texture [7]. Ionic conductivity was obtained by fitting Eq. (1) to the experimental data for planar and homeotropic configuration of the samples. Figure 4 illustrates temperature dependence of ionic conductivity components $\left(\sigma_{\|}\right.$and $\left.\sigma_{\perp}\right)$. It is interesting that for $5 \mathrm{OSCl}$ the ionic conductivity along the director in nematic phase is an activation process, whereas for $6 \mathrm{OSCl}$ is non-activation one. Such behavior can result from different types of ions, which take part in conductivity process for $5 \mathrm{OSCl}$ than for $6 \mathrm{OSCl}$.

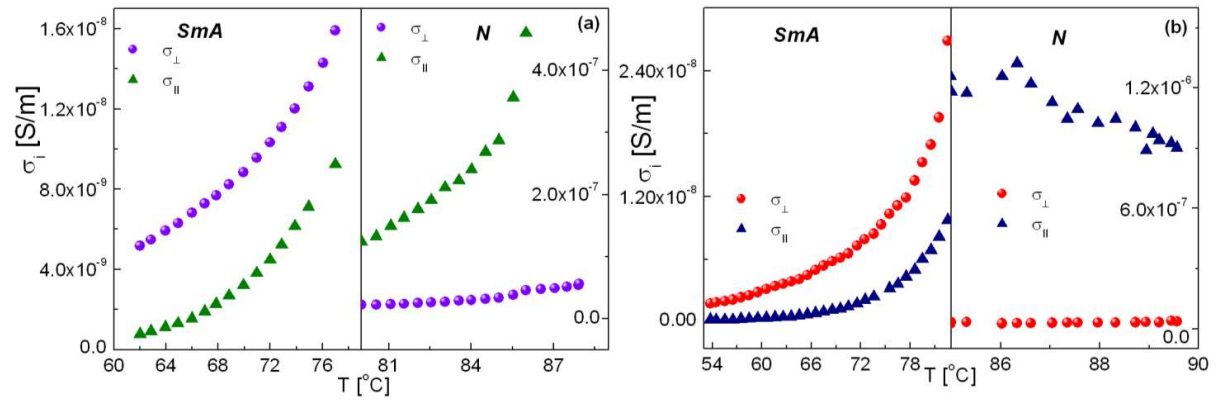

Fig. 4. Temperature dependence of ionic conductivity obtained for planar and homeotropic alignment for $5 \mathrm{OSCl}$ (a) and $6 \mathrm{OSCl}(\mathrm{b})$.

One can also define conductivity anisotropy as

$$
\Delta \sigma=\sigma_{\|}-\sigma_{\perp}
$$

where $\sigma_{\|}$and $\sigma_{\perp}$ are conductivities for homeotropic and planar alignment of the sample, respectively. 


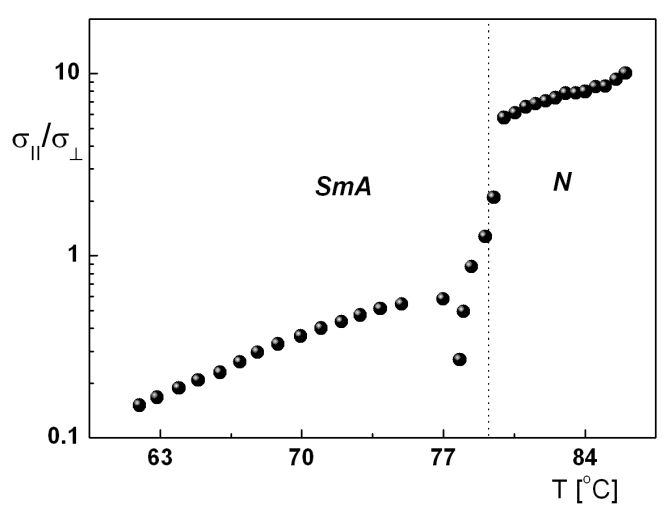

Fig. 5. A ratio of parallel and perpendicular components of ionic conductivity versus temperature for $5 \mathrm{OSCl}$.

From Figs. 4 and 5 one can conclude that for both substances studied the anisotropy of conductivity is positive in the nematic phase and negative in $\mathrm{SmA}$ phase. It means that in nematic phase the ionic conductivity is higher along the director, whereas in SmA phase the diffusion of ions is easier within the smectic layers in $(x, y)$ planes of the $\mathrm{SmA}_{2}$ phase. The ratio of conductivities $\sigma_{\|} / \sigma_{\perp} \approx 0.2$ is typical of biological membranes.

\section{Conclusions}

Summing up, the dielectric measurements done in the frequency range from $40 \mathrm{~Hz}$ to $15 \mathrm{MHz}$, allowed to determine the dielectric and conductivity anisotropy in liquid crystalline phases of strongly polar thioesters. For both substances, one molecular relaxation process was observed for homeotropic alignment, and it is interpreted as the reorientation of molecules around short molecular axis. Dielectric anisotropy is positive in both phases of two studied compounds. However, it decreases with decreasing temperature which is typical of the systems with strong dipole-dipole correlations. On the other hand, ionic conductivity $(\Delta \sigma)$ changes the sign at the transition from $\mathrm{SmA}$ to $\mathrm{N}$ phase. In SmA phase $\Delta \sigma$ is negative, which means that the conductivity within smectic layers (planar alignment of molecules) is higher than crosswise the smectic layers (homeotropic configuration).

\section{References}

[1] J.R. Willmott, C.J. Davenport, J. Newton, H.J. Coles, Mol. Cryst. Liq. Cryst. 343, 209 (2005).

[2] D.J. Gardiner, H.J. Coles, J. Appl. Phys. 100, 124903 (2006).

[3] E.F. Carr, W.T. Flint, J.H. Parker, Phys. Rev. A 11, 1732 (1975).

[4] E. Kochowska, S. Németh, G. Pelzl, Á. Buka, Phys. Rev. E 70, 011711 (2004).

[5] S. Naemura, A. Sawada, Mol. Cryst. Liq. Cryst. 400, 79 (2003). 
[6] M.D. Ossowska-Chruściel, P. Roszkowski, A. Rudzki, J. Chruściel, Liq. Cryst. 32, 877 (2005).

[7] A. Mikułko, M. Fraś, M. Marzec, M.D. Ossowska-Chruściel, J. Chruściel, S. Wróbel, unpublished results.

[8] L. Longa, W.H. De Jeu, Phys. Rev. A 28, 2380 (1983).

[9] J.D. Bunning, T.E. Faber, P.L. Sherell, J. Phys. (France) 42, 1175 (1981).

[10] M.D. Ossowska-Chruściel, A. Mikułko, I. Karkuszewska, S. Wróbel, J. Chruściel, M. Marzec, W. Haase, in: Neutron Scattering and Complementary Methods in Investigations of Condensed Phase, Eds. J. Chruściel, A. Szytuła, W. Zając, Univ. of Podlasie Publishing House, Siedlce 2005, p. 200. 